\title{
COMPARISON FOR WIMAX SCHEDULING ALGORITHMS AND PROPOSAL QUALITY OF SERVICE IMPROVEMENT IN WIMAX NETWORKS
}

\author{
${ }^{1}$ R. Nandhini and ${ }^{2}$ N. Devarajan \\ ${ }^{1}$ Department of Computer Science, \\ Faculty of Information and Communication Engineering, Anna University, Chennai, 600025, Tamilnadu, India \\ ${ }^{2}$ Department of Electrical Engineering, Government College of Technology, Coimbatore, 641013, Tamilnadu, India
}

Received 2013-07-03, Revised 2013-08-18; Accepted 2013-11-19

\begin{abstract}
The objective of the broadband wireless technologies is to ensure the end to end Quality of Service (QoS) for service classes. Wimax is a revolution in wireless networks which could support real time multimedia services. In order to provide QoS support and efficient usage of system resources an intelligent scheduling algorithm is needed. The design of detailed scheduling algorithm is a major focus for researchers and service providers. In this study, a channel aware cross-layer scheduling algorithm for Wimax networks has been proposed. This scheme employs Signal to Noise Ratio (SNR) value which allocates bandwidth based on the information about the quality of the channel and service requirements of each connection. The proposed algorithm is described in detail and evaluated through series of simulation. The simulation results prove that the proposed algorithm reduces the packet loss rate and delay and thus improves throughput by $12.8 \%$.
\end{abstract}

Keywords: Cross-Layer, IEEE 802.16, QoS, Scheduling, Wimax

\section{INTRODUCTION}

Wimax (World Wide Interoperability for Microwave Access) is an IEEE standard (IEEE $802.16 \mathrm{~d} / \mathrm{e}$ ) that promises high bandwidth solution with long range for metropolitan area networks. IEEE 802.16 is able to cover large geographical area since the distance between the Base Station (BS) and the Subscriber Station (SS) can extend up to 30 miles (Mai et al., 2010). IEEE 802.16 defines the layer 1 (Physical (PHY)) and layer 2 (Data link or Media Access Control (MAC)) of the Open System Interconnection (OSI) seven layer network model. The different types of standards for PHY supports are Single Carrier (SC), Single Carrier Access (SCA), Orthogonal Frequency Division Multiplexing (OFDM) and Orthogonal Frequency Division Multiple Access (OFDMA). Recent researches focus mainly on the OFDM and OFDMA PHY supports. These standards define two operational modes for communication namely; mesh mode and point-to-multipoint mode. In mesh mode, the SSs can communicate with each other and also with the BS. In point-to-multipoint mode, SSs are supposed to communicate only through BS. BS has dedicated buffers and slots for downlink connection. During uplink, slots are allotted per SS and not per connection. Uplink channel is shared by all SSs, whereas downlink channel is used only by BS (TCS, 2009).

The MAC layer functions (Rengaraju et al., 2010) of IEEE 802.16e are described in Fig. 1. Internet Protocol (IP), Ethernet and Asynchronous Transfer Mode (ATM) traffic are supported by convergence sublayer. This layer converts the traffic into MAC data units. Wimax network provides broadband access for services having different QoS requirements and different traffic priorities. It is the responsibility of the MAC layer to schedule the traffic flows and to allocate the bandwidth such that QoS requirements of each flow are satisfied.

Corresponding Author: R. Nandhini, Department of Computer Science, Faculty of Information and Communication Engineering, Anna University, Chennai, 600025, Tamilnadu, India 
MAC layer

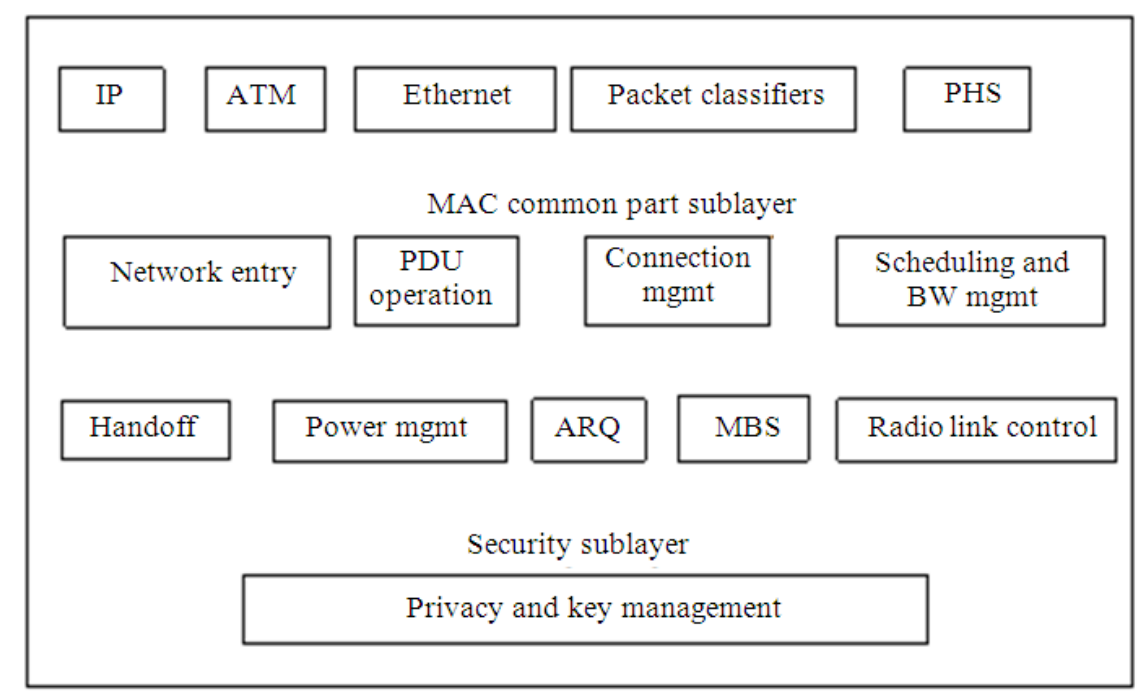

PHS layer

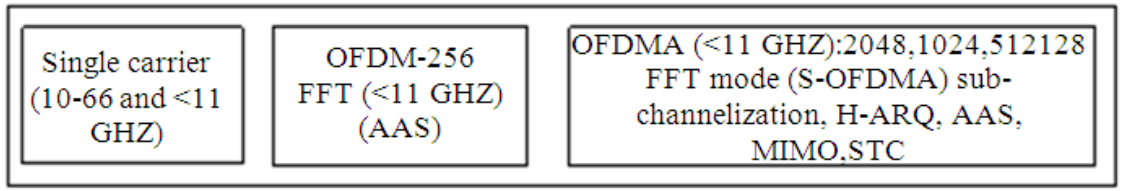

Fig. 1. IEEE 802.16e-2005 protocol stack (Rengaraju et al., 2010)

IEEE 802.16e is expected to provide QoS for fixed and mobile users. QoS depends upon a number of implementation details like scheduling, buffer management and traffic shaping. The responsibility of scheduling and BW management is to allocate the resources efficiently based on the QoS requirement of the service classes. There are five service classes which are defined in IEEE802.16e standard. They are as follows:

- Unsolicited Grant Services (UGS): Designed to support Constant bit rate services like voice applications

- $\quad$ Real Time Data Polling Services (RTPS): Designed to support real time services that generates variable size data packets on a periodic basis like MPEG but insensitive to delay

- Extended Real Time Polling Services (ERTPS): Designed to support real time applications with variable data rates which require guaranteed data and delay. Example: Voice Over Internet Protocol (VOIP) with silence suppression

- $\quad$ Non Real Time Polling Services (NRTPS): Designed to support non real time and delay tolerant services that require variable size data grant burst types on a regular basis such as File Transfer Protocol (FTP)

- Best Effort (BE): Designed to support data streams that do not require any guarantee in QoS such as Hyper Text Transfer Protocol (HTTP)

The QoS provision in Wimax requires complete scheduling mechanism which is not defined in the standard. The scheduling mechanisms have to provide guarantee to the bandwidth required by SS as well as wireless link usage. The goal of designing a scheduler is to minimize power consumption and Bit Error Rate (BER) and to maximize the total throughput. Wired networks scheduling algorithms are unfit for wireless networks due to location dependency and burst channel errors. Thus, the scheduling algorithm should take Wimax QoS classes and service requirements into consideration.

The rest of the study is organized in the following way. Section 2 is the survey about related existing work. Section 3 describes about the basic architecture of IEEE 802.16 e standard. Section 4 shows working nature of scheduling algorithm. Section 5 shows the proposed scheduling algorithm. Simulation results have been 
presented in Sections 6. The conclusion and future extension of the study is explained in Section 7.

\section{LITERATURE REVIEW}

Borin and Fonseca (2009) proposed a standard compliant scheduling solution for uplink traffic in IEEE 802.16 networks but wireless channel characteristics are not considered in this solution. Different schedulingalgorithms has beencompared in (Arhaif, 2011) and evaluated using Qualnet 5.0. The Diffservenabled (DIFFserv), Round Robin (RR), Self Cloacked Fair (SCF), Strict Priority (SP), Weighted Round Robin (WRR) are scheduling algorihtms compared by authors. In other hand, (Mardini et al., 2011) WiMAX technology based on IEEE 802.16 standard which is a Broadband Wireless Access (BWA) that offers mobile broadband connectivity. But none of them is able to support QoS requirements of the five types of service flow defined by the IEEE 802.16e standard. Some of the past research works uses a history of packet delays to classify packets in four classes and the scheduler gives higher priority to packets destined to users whose instant channel conditions are better A study on centralized scheduling for Unsolicited Grant Service and Real-time Polling Service has been presented by Goyal and Sahoo (2010). The proposed scheduling mechanism meets the quality of service for classes which is discussed by author. Since real time services need extra bandwidth for variable data changing rate, it increases the performance by reducing delay and loss rate. It has been proved that the scheduling algorithm that considered wireless link perform better than the algorithm that does not consider wireless link (Revankar et al., 2010). Chuang et al. (2013) propose a QoS scheme based on Modified Deficit Round Robin (MDRR) of packet scheduling and Call Admission Control (CAC) with the channel condition for non-realtime service. H.264/AVC is now the standard for video streaming because of its high compression efficiency, robustness against errors and network-friendly features. However, providing the desired quality of service or improving the transmission efficiency for H.264 video transmissions over wireless networks present numbers of challenges. The author (Hsiao et al., 2011) consider those challenges and survey existing mechanisms based on the protocol layers they work on. Finally, they address some open research issues concerning for H.264 video transmission in wireless networks and (Ghazizzadeh et al., 2009) it is estimated according to the instantaneous transmission rate. Fluid Fair Queuing (FFQ) is a well-known algorithm which provides fairness among the packets through the shared link (TCS, 2009). TCS (2009), the author classified the uplink schedulers as Weighted Round Robin (WRR), Earliest Dead line First (EDF) and Weighted Fair Queuing (WFQ). Down link schedulers are classified into Proportional Fairness (PF), Adaptive Proportional Fairness (APF), Integrated Cross-Layer (ICL) and Round Robin (RR).

Revankar et al. (2010), the authors emphasis the MAC scheduling architecture for IEEE 802.16 wireless networks in both uplink and downlink direction to broadcast the frame. Further they used WFQ as uplink as well as downlink scheduling algorithm for improving delay and throughput. There is no separate scheduling policy for Unsolicited Grant Services (UGS). Even though there are vast number of works based on scheduling in single hop networks, these algorithms cannot be applied for multihop relay scenarios.

TCP aware uplink scheduling algorithm focuses on the allocation of bandwidth higher than actual sending rate of the connection. Comparative analysis of different QoS algorithm in Wimax) is shown in Table 1.

\section{IEEE 802.16 SCHEDULING ARCHITECTURE}

The basic IEEE 802.16 architecture (Jain and Verma, 2008) includes Base station and multiple Subscriber Stations (SS). Both base station and subscriber station are immobile when client wants to connect SS to a mobile station. Base station acts as a central entity which transfers all the data from the subscriber stations in point-to-multi point architecture. Two or more subscribers are not allowed to communicate directly. The BS and SS architecture are connected through wireless links. Communication occurs in two directions: From BS to $\mathrm{SS}$ is called downlink and from SS to BS is called uplink. During downlink, BS broadcasts data to all subscribers and subscriber selects packets destined for it. Uplink channel is shared between all multiple SSs while downlink channel is used only by BS.

In order to ensure slotted channel sharing and the slots are allocated by BS to various SS in one uplink frame, Time Division multiplexing (TDD) or Frequency Division multiplexing (FDD) is used. This slot allocation information is broadcast by BS through the uplink map message (UL-MAP) at the beginning of each frame. ULMAP contains information element which includes the transmission opportunities and the time slots in which the SS can transmit during the uplink subframe. 
Table 1. Scheduling algorithms comparison

\begin{tabular}{|c|c|c|}
\hline Algorithm & Advantage & Disadvantage \\
\hline Proportional Fair algorithm & $\begin{array}{l}\text { Fairness in scheduling } \\
\text { Priority based, Simple }\end{array}$ & No QoS Guarantee \\
\hline Cross-layer Scheduling algorithm & $\begin{array}{l}\text { QoS guarantee } \\
\text { Channel quality is considered in } \\
\text { scheduling }\end{array}$ & $\begin{array}{l}\text { Complex implementation } \\
\text { Slots are allocated to higher priority } \\
\text { connection }\end{array}$ \\
\hline $\begin{array}{l}\text { TCP aware uplink scheduling } \\
\text { algorithm }\end{array}$ & $\begin{array}{l}\text { Efficient utilization of resources } \\
\text { among BE connection }\end{array}$ & $\begin{array}{l}\text { Complex implementation handle only one } \\
\text { class service }\end{array}$ \\
\hline $\begin{array}{l}\text { Cross-layer scheduling for } \\
\text { OFDMA networks }\end{array}$ & Improved packet loss rate, delay & $\begin{array}{l}\text { Spectral efficiency of system degrades about } \\
0.3 \mathrm{bps} / \mathrm{Hz} \text {. }\end{array}$ \\
\hline Cross layer downlink scheduling & $\begin{array}{l}\text { Scheduling all services flow types } \\
\text { Good throughput } \\
\text { High Frame utilization }\end{array}$ & Can be implemented only at the base station \\
\hline EDF & Focusing on efficiency & Unfit for non real time applications \\
\hline WRR & Suitable for non-real time applications & Does not perform well in variable packet size \\
\hline $\begin{array}{l}\text { Enhanced Cross-layer downlink } \\
\text { scheduling algorithm }\end{array}$ & $\begin{array}{l}\text { Fairness } \\
\text { Guarantee to real and non-real time } \\
\text { connection }\end{array}$ & Subscriber mobility is not considered \\
\hline $\begin{array}{l}\text { Cross layer designed scheduling } \\
\text { (Rengaraju et al., 2010) algorithm for } \\
\text { Wimax uplink (DMIA) }\end{array}$ & $\begin{array}{l}\text { Meets all QoS requirement of all } \\
\text { service classes higher throughput, } \\
\text { lower delay, jitter and packet loss rate }\end{array}$ & \\
\hline
\end{tabular}

\section{SCHEDULING ALGORITHMS}

IEEE 802.16 MAC layer adopts a connection oriented architecture in which a connection must be established before data communications. Each connection is assigned a unique identifier (connection IDI) and it is associated with a service flow which defines the desired QoS level of the connection. In a standard scheduling framework, data packets arriving at the BS are classified into connections which are then classified into service flows. Packets of same service flow are placed in a queue and then further classified based on their service priorities of the connection. For packets in multiple queues with different service requirements, a packet scheduler is employed to decide the service order of the packets from the queues. If properly designed a scheduling algorithm may provide the desired service guarantees.

The scheduler should consider the following important parameters:

- The traffic service type

- The set of QoS requirements of the connections

- The capacity of bandwidth for data transmission

- The bandwidth requirements from the connections

- Waiting time of bandwidth request in the system

The ideal scheduler should be able to make optimum use of the available bandwidth to reduce traffic delays and satisfy the QoS requirements to the best extent so as to reduce packets drop rate and sustain the QoS support.
Wimax schedulers can be classified into two main categories, channel unaware schedulers where the channels are assumed to be error free and channel aware schedulers where channel state information is taken into consideration while scheduling the packet. Channel unaware schedulers are further classified into homogeneous and hybrid schedulers. Hybrid schedulers combine more than one scheduler to satisfy the QoS requirements of the multiple service class traffic in Wimax networks.

Figure 2 represents the cross-layer scheduler methodology. WRR, WFQ, EDF, Strict Priority (SP) are the few examples of homogeneous scheduling algorithms. According to the research, none of the homogeneous scheduling algorithm provides the QoS requirement of Wimax networks. So, researchers attempted to hybrid the algorithms to get a satisfied QoS level. Cross-layer scheduling is one of the algorithms in channel aware scheduling algorithm.

\section{PROPOSED CROSS-LAYER SCHEDULING ALGORITHM}

The main focus of the cross layer design is to provide best possible end-to-end performance for the applications. The objective is to maximize the total throughput when satisfying the QoS requirements of different service classes. The proposed scheduling algorithm modifies cross-layer algorithm which incorporates SNR value and the minimum required throughput of the $\mathrm{SS}$ in its formulation. The SS with highest priority is selected to transmit in the frame. The priority of the SS is calculated based on the traffic class it belongs to. 


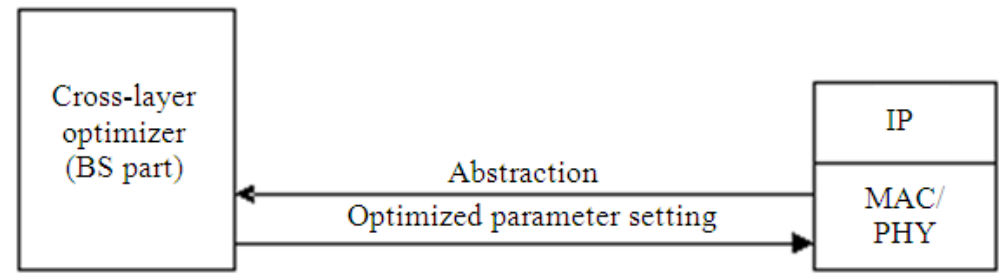

\begin{tabular}{|c|c|c|}
\hline Application & \multirow{4}{*}{ Optimized parameter setting } & \multirow{5}{*}{$\begin{array}{c}\text { Cross-layer } \\
\text { optimizer } \\
\text { (SS part) }\end{array}$} \\
\hline RTP & & \\
\hline UDP/IP & & \\
\hline \multirow[t]{2}{*}{$\mathrm{MAC} / \mathrm{PHY}$} & & \\
\hline & & \\
\hline
\end{tabular}

Fig. 2. Cross-layer functionality

\section{Algorithm:}

1. Define higher priority queue

2. Schedule the Bandwidth request opportunities which should be scheduled in next frame

3. Periodically check the deadline for the service flow

4. Do check the bandwidth minimum availability

5. Resources should be periodically distributed among the service flow according to the deadline

The algorithm is executed at the BS at the beginning of every frame thereby priority is assigned to each SS. The cross layer algorithm proposed in (El-Fishawy et al., 2011) implies three drawbacks. The modified cross-layer scheduling algorithm improves those drawbacks in the following ways and efficiently manages the bandwidth allocation:

- Required slots are allocated to higher priority packets and not only to one packet

- Multiple packets are in same priority, the one with earliest arrived has been picked up to decrease the delay

- Fragmentation is done for service types to make use of the available slots except the ertPS connection in Wimax frame

Based on SNR, the type of modulation can be chosen from Table 2 (Shuaibu et al., 2010).
Table 2. MCS and receiver SNR

\begin{tabular}{lllc}
\hline S/N & Modulation & Coding rate & SNR $(\mathrm{dB})$ \\
\hline 1 & QPSK & $1 / 2$ & 5.0 \\
& & $3 / 4$ & 8.0 \\
2 & 16-QAM & $1 / 2$ & 10.5 \\
& & $3 / 4$ & 14.0 \\
3 & \multirow{2}{*}{ 64-QAM } & $1 / 2$ & 16.0 \\
& & $2 / 3$ & 18.0 \\
& & $3 / 4$ & 20.0 \\
\hline
\end{tabular}

Four different buffers were used, each for one service flow. Each buffer has length $t$ and each packet received in the uplink session is stored in the identification, SNR, arrival time and packet size. The responsibility of the scheduler is to visit each buffer during the downlink subframe and to schedule the packets based on the proposed algorithm.

\section{PERFORMANCE EVALUATION}

\subsection{Simulation Platform}

The scheduler proposed in this study was implemented in the IEEE 802.16 module in Network Simulator (NS-2) simulator. The ns 2 is a widely used tool for the simulation of packet switched networks. It gives huge support for simulation of TCP routing and Mac protocols over wired and wireless networks. Network elements in ns 2 simulator are developed as classes in object oriented manner. It has Object Tool Command Language (OTCL) interpreter for easy user interface, has input models which is written in 
Tool Command Language (TCL) scripts. A base station and a subscriber station can be set up as a node in ns 2 . When the number of nodes increases the amount of packets received and sent increases. For a single node configuration the simulation would run fairly. But as the number nodes increases the packet traffic will arise.

The simulated network uses a Point to Multipoint topology (PMP) with a centralized BS and the SS. The distance between MSS and BS ranges from 1600 to 1800 meters. In our simulation, for sending the bandwidth request from all SSs, unicast polling is used. Here, the Grant per Subscriber Station (GPSS) bandwidth allocation scheme is used. In the simulation, number of calls generated by SSs is varied and is randomly generated.

\subsection{Simulation Parameters}

The simulated network uses a Point to Multipoint topology (PMP) with a centralized BS and the SS. The distance between MSS and BS ranges from 1600 to 1800 $\mathrm{m}$. In our simulation, for sending the bandwidth request from all SSs, unicast polling is used. Here, the Grant per Subscriber Station (GPSS) bandwidth allocation scheme is used. In the simulation, number of calls generated by SSs is varied and is randomly generated.

The simulation parameters settings are shown in Table 3. Base station receives all transmitted packets from the subscriber stations; assigns packet serial number, packet service flow identification and arrival time and stores the packet in appropriate buffer of the service flow. Each transmitted packets have its own estimated SNR value as shown in Table 2. BS schedules the packets based on the cross-layer scheduling algorithm during the downlink session. According to the values of packet size and SNR value, required numbers of slots are allotted for each of the packets. If the required number of slots on the current frame is not enough to schedule the current packet, then the packet is lost. The buffers are used for handling different service flow. Each buffer can store 250 packets at a time. If the buffer is full and there is a packet on the queue the packet is considered to be lost since there is no memory to hold it. Once the packet is scheduled, it should be removed from the buffer and memory is considered empty to store the next packet. The uplink duration is $4.5 \mathrm{~ms}$ and the downlink duration is $5.3 \mathrm{~ms}$.

\subsection{Simulation Results}

The experiment was conducted with the proposed algorithm with three different service flows. The vital
QoS parameters throughput, packet loss, average delay was calculated for three different kinds of service flow with varied number of SSs. To analyze the QoS in Wimax networks, VOIP application is considered. For each of the scenario, the simulation time is $40 \mathrm{~s}$. The following simulation results are obtained based on average of 10 independent simulations presented in $95 \%$ confidence intervals.

For the codec scheme G.711, the number of nodes with the VOIP traffic is varied from 1, 3, 5, 7, 9 and 11 . The experiment is repeated only for the following service flows defined by IEEE 802.16e standards BE, rtPS and UGS.

\subsubsection{Throughput}

Throughput is defined as the measure of data rate (bits per second) generated by the application. To calculate throughput the size of each packet was added. The total time was calculated by the difference between the time that the first packet started and the time that the last packet reached the destination. Data collected from all three service flows for throughput are presented in a single chart. Since the UGS traffic has less packet loss the throughput is high. The throughput of rtPS and BE are very similar. UGS service flow is designed with constant bit rate traffic, in which periodic bandwidth is allocated by BS to SS. As we can see from Fig. 3, the graph shows the throughput of all three service flow for cross-layer scheduling algorithm.

\subsubsection{Packet Loss}

Packet loss is the sum of all the packets which do not reach the destination over the sum of packets which leaves the destination. The ratio of total data sent to total data lost gives the packet loss.

The comparative packet loss percent variation is shown in the Fig. 4. Since UGS traffic support real time traffic, it has very low packet loss. This is one of the expected behaviors. In case of rtPS, SS was allocated with fixed bandwidth and transmits the data packets in a specific slot. Bandwidth is not allotted for rtPS service flow on regular basis. So the packet loss is comparatively low with BE service flow.

\subsubsection{Average Delay}

The time taken by the packets to start from the source and reach the destination and traverse back to source is the delay produced by packet. The source which causes the delay can be propagation delay, network delay, source delay, destination delay. 
R. Nandhini and N. Devarajan / American Journal of Applied Sciences 11 (1): 8-16, 2014

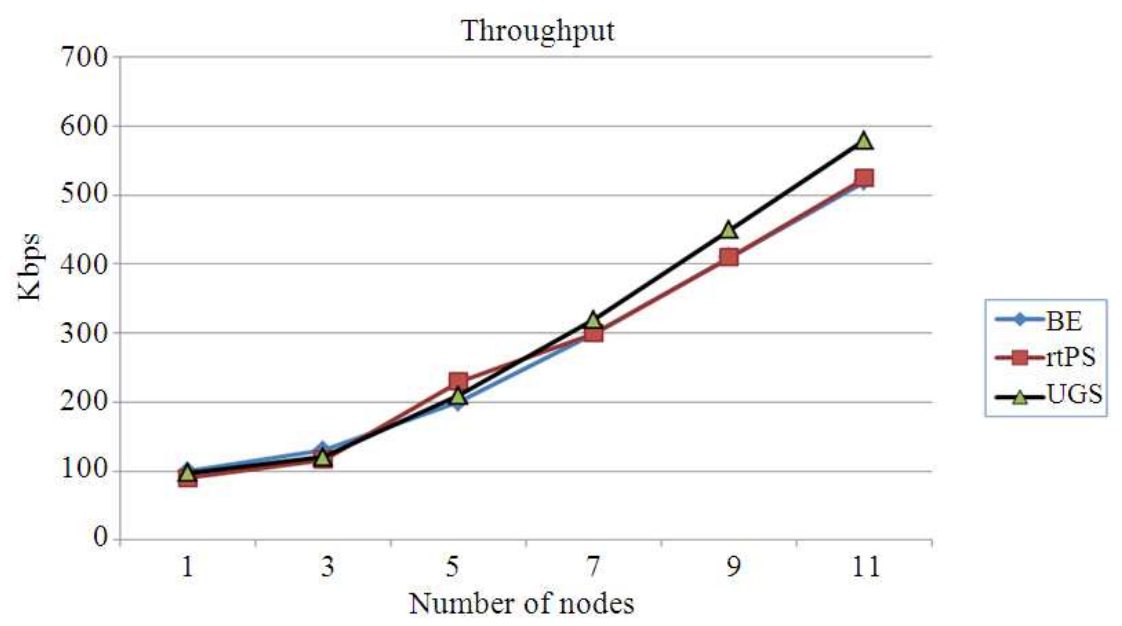

Fig. 3. Throughput with the number of nodes for all three service flow



Fig. 4. Packet loss with the number of nodes for all three service flow

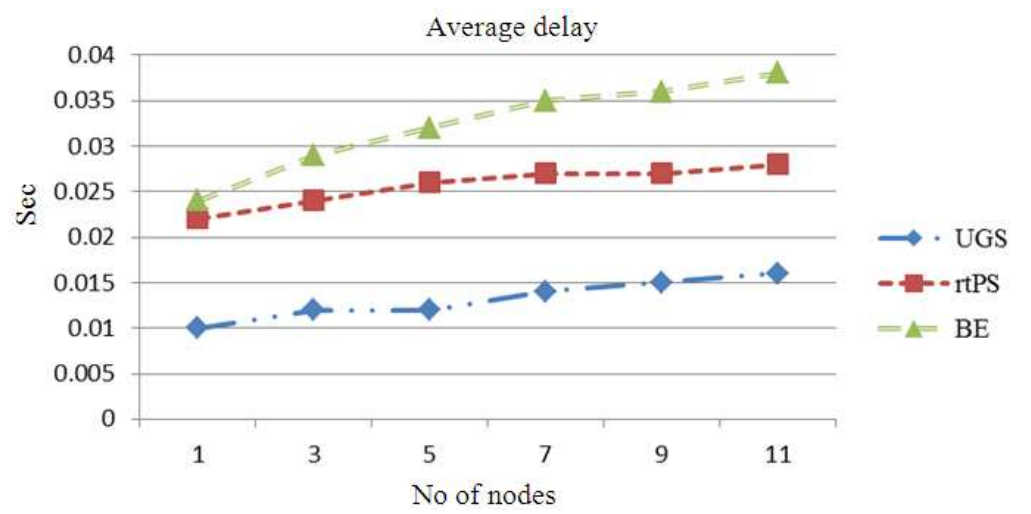

Fig. 5. Average delay with the number of nodes for all three service flow 
Table 3. System parameters

\begin{tabular}{ll}
\hline Parameter & Value \\
\hline $\begin{array}{l}\text { Physical layer } \\
\text { No of OFDM symbols } \\
\text { and sub channels }\end{array}$ & $\begin{array}{l}\text { Wireless MAN-OFDM,TDD } \\
\text { Bandwidth and frame } \\
\text { duration }\end{array}$ \\
$\begin{array}{l}\text { Minimum resource } \\
\text { allocation unit(slot) }\end{array}$ & $10 \mathrm{MHz}$ and $5 \mathrm{~ms}$ \\
Max PDU size & $\begin{array}{l}\text { 2 OFDM symbols in time, } \\
1 \text { sub channel in frequency }\end{array}$ \\
\hline
\end{tabular}

Table 4. Aggregate parameters for all service flow

\begin{tabular}{lrrr}
\hline Algorithm & \multicolumn{1}{c}{ UGS } & \multicolumn{1}{c}{ rtPS } & \multicolumn{1}{c}{ BE } \\
\hline Throughput (Mbps) & 292.1250 & 278.500000 & 276.66670000 \\
Packet loss (\%) & 0.0472 & 0.047760 & 0.72290000 \\
Average delay (ms) & 0.0395 & 0.051333 & 0.032333333 \\
\hline
\end{tabular}

Three service flow average delay variation was comparatively shown in Fig. 5. The delay for UGS service flow and rtPS service flow are close to each other which is shown in Fig. 5. BE service flow has highest delay when compared with other 2 service flows.

From Table 4, it is proved that UGS service flow has higher throughput, lowest delay and lowest packet loss. This makes UGS traffic a most suitable service flow for VOIP traffic.

\section{CONCLUSION}

In this study we addressed the problem of a crucial scheduling strategy which takes channel condition as a feedback for better bandwidth usage for IEEE 802.16 wireless networks. In this study, static IEEE 802.16 network is considered. To validate the proposed algorithm a Wimax simulation platform based on NS2 has been implemented. The simulation results have verified that our proposed scheduling algorithm is capable to enhance the performance of Wimax networks. The performance improvement of the proposed scheme is illustrated through the simulation results. The proposed algorithm not only meets all the QoS requirements of the service classes but also provides higher throughput, low delay and packet loss rate, while promises the fairness among all the other service class. Currently we worked on the VOIP codec scheme and three service classes along with the proposed scheduling scheme. In the future work, subscriber mobility will be considered and more codec schemes for VOIP will be taken for more real-time operating environment.

\section{REFERENCES}

Arhaif, M.S., 2011. Comparative study of scheduling algorithms in WiMAX. Int. J. Scient. Eng. Res., 2: 83-89.

Borin, J.F. and N.LS.D. Fonseca, 2009. Uplink scheduler and admission control for the IEEE 802.16 standard. Proceedings of the IEEE Global Telecommunications Conference, Nov. 30-Dec. 4, IEEE Xplore Press, Globecom, pp: 1-6. DOI: 10.1109/GLOCOM.2009.5425779

Chuang, H.S., L.T. Lee and C.F. Wu, 2013. A crosslayer QoS scheme of non-real-time service in WiMAX. Int. J. Future Generat. Commun. Network., 6: 87-98.

El-Fishawy, N.A., M. Zahra, M. Ebrahim and M.M. ElGamala, 2011. Modified cross-layer scheduling for mobile WiMAX networks. Proceedings of the 28th National Radio Science Conference, Apr. 26-28, IEEE Xplore Press, Cairo, pp: 1-10. DOI: 10.1109/NRSC.2011.5873602

Ghazizzadeh, R., P. Fan and Y. Pan, 2009. A two layer channel aware scheduling algorithm for IEEE 802.16 broadband wireless access systems. J. Applied Sci., 9: 449-458. DOI: 10.1109/MNET.2009.4305171

Goyal, P. and A. Sahoo, 2010. A scheduling and call admission control algorithm for WiMax mesh network with strict QoS guarantee. Proceedings of the 2nd International Conference on Communication Systems and Networks, Jan. 5-9, IEEE Xplore Press, Bangalore, $\quad$ pp: 1-10. DOI: 10.1109/COMSNETS.2010.5431997

Hsiao, Y.M., J.F. Lee, J.S. Chena and Y.S. Chu, 2011. H.264 video transmissions over wireless networks: Challenges and solutions. Comput. Commun., 34: 1661-1672. DOI: 10.1016/j.comcom.2011.03.016

Jain, A. and A.K. Verma, 2008. Comparative analysis of scheduling algorithms for wimax. Proceedings of the National Conference on Mobile and Pervasive Computing, (MPC' 08), Thapar University, Patiala.

Mai, Y.T., C.C., Yang and Y.H. Lin, 2010. Design of the cross layer qos framework for IEE 802.16 PMP networks. IEICE Trans. Commun., E91: 1360-1369. DOI: $10.1109 /$ TVT.2006.873832 
Mardini, W., Y. Khamayseh, M.B. Yasin and G. Obiedat, 2011. Survey of latest WiMAX technologies and techniques. Int. Rev. Comput. Softw.

Rengaraju, P., C.H. Lung and A. Srinivasan, 2010. QoS assured uplink scheduler for wimax networks. Proceedings of the IEEE 72nd Vehicular Technology Conference Fall, Sep. 6-9, IEEE Xplore Press, Ottawa, ON., pp: 1-5. DOI: 10.1109/VETECF.2010.5594592
Revankar, P.S., A.S. Kapse and W.Z. Gandhare, 2010. Overview of MAC scheduling algorithm for IEEE 802.16 wireless networks. Int. J. Comput. Applic. DOI: $10.5120 / 689-968$

Shuaibu, D.S., S.K. Syed-yusof and N. Fisal, 2010. A cross layer approach for packet scheduling at downlink of wimax IEEE 802.16e. Eur. J. Scient. Res., 45: 529-539.

TCS, 2009. WiMAX base station scheduling algorithms. Tata Consultancy Services. 